\title{
Assessment of the effects of hepatic impairment and smoking on the pharmacokinetics of a single oral dose of the soluble guanylate cyclase stimulator riociguat (BAY 63-2521)
}

\author{
Reiner Frey, ${ }^{1}$ Corina Becker, ${ }^{1}$ Sigrun Unger, ${ }^{2}$ Anja Schmidt, ${ }^{1}$ Georg Wensing, ${ }^{1}$ Wolfgang Mück ${ }^{1}$ \\ 'Clinical Pharmacology, Pharma Research Center, Bayer Pharma, Wuppertal, Germany; ${ }^{2}$ Global Biostatistics, Pharma Research Center, Bayer Pharma, \\ Wuppertal, Germany
}

\begin{abstract}
Riociguat, a soluble guanylate cyclase stimulator developed for the treatment of pulmonary hypertension, is metabolized in part by the liver. Expression of one of the metabolizing enzymes, CYP1A1, is induced by aromatic hydrocarbons in tobacco smoke. Two nonrandomized, nonblinded studies were conducted to investigate the pharmacokinetics of riociguat in individuals with mild (Child-Pugh A) or moderate (Child-Pugh B) hepatic impairment associated with liver cirrhosis compared with that in age-, weight-, and sex-matched healthy controls: study 1 included smokers and nonsmokers, and study 2 included nonsmokers only. Data from these studies were integrated for analysis. All participants $(N=64)$ received a single oral dose of riociguat $1.0 \mathrm{mg}$. Riociguat exposure was significantly higher in individuals with Child-Pugh B hepatic impairment than in healthy controls (ratio: 153\% [90\% confidence interval: 103\%-228\%]) but was similar in those with Child-Pugh A hepatic impairment and controls. The half-life of the riociguat metabolite M1 was prolonged in patients with Child-Pugh B or A hepatic impairment compared with that in controls by approximately $43 \%$ and $24 \%$, respectively. Impaired hepatic function was associated with higher riociguat exposure in nonsmokers compared with the population of smokers and nonsmokers combined. Riociguat's safety profile was similar in individuals with impaired or normal liver function. In conclusion, moderate hepatic impairment was associated with increased riociguat exposure compared with that in controls, probably as a result of reduced clearance of the metabolite M1. This suggests that dose titration of riociguat should be administered with particular care in patients with moderate hepatic impairment.
\end{abstract}

Keywords: pulmonary hypertension, clinical pharmacology, cirrhotic, Child-Pugh, CYP1A1.

Pulm Circ 2016;6(S1):S5-S14. DOI: 10.1086/685015

Pulmonary hypertension $(\mathrm{PH})$ is caused by excessive vasoconstriction and the proliferation of smooth muscle and endothelial cells within the small pulmonary arteries, leading to remodeling of the pulmonary vasculature. The resulting sustained rise in pulmonary arterial pressure increases the workload of the right ventricle, which can lead to right ventricular heart failure. Without specific treatment, $\mathrm{PH}$ is a progressive and debilitating condition with a poor prognosis. Twenty-five years ago, the median survival from the time of diagnosis was only 2.8 years for those with idiopathic pulmonary arterial hypertension (PAH). ${ }^{1}$ Although advances in therapies have improved outcomes, the estimated 7-year survival rate from diagnosis of idiopathic or familial PAH remains less than $60 \%{ }^{2}$

In healthy lungs, the balanced activity of vasoconstrictive agents (including thromboxane A2 and endothelin) and vasodilatory agents (including nitric oxide [NO] and prostacyclins) ensures that the pulmonary vasculature is kept in a low-pressure state. ${ }^{3}$ In $\mathrm{PH}$, this balance is disrupted: the availability of vasodilators is reduced and production of endothelin is increased. ${ }^{4}$ Stimulation of the NO receptor, soluble guanylate cyclase (sGC), by $\mathrm{NO}$ increases production of the vasodilatory second messenger cyclic guanosine monophosphate (cGMP). ${ }^{3,5}$ Upregulation of sGC expression has been observed in the smooth muscle cells of patients with $\mathrm{PH}$ and in animal models of $\mathrm{PH}$, suggesting that $\mathrm{sGC}$ is an attractive target for pharmacotherapy. ${ }^{6}$ Indeed, preclinical studies indicate that right heart hypertrophy and lung vascular remodeling can be reversed through stimulation of sGC. ${ }^{6}$

Riociguat (BAY 63-2521) is the first oral sGC stimulator developed for the treatment of $\mathrm{PH}^{7}$ The drug increases cGMP production via a novel dual mode of action involving direct stimulation of sGC in an NO-independent manner and an increase in the sensitivity of sGC to low levels of NO. ${ }^{7}$ In clinical studies, including two phase 3 trials (Chronic Thromboembolic Pulmonary Hypertension Soluble Guanylate Cyclase-Stimulator Trial 1 [CHEST-1] and Pulmonary Arterial Hypertension Soluble Guanylate Cyclase-Stimulator Trial 1 [PATENT-1]), oral riociguat was readily absorbed, exhibited dose-proportional pharmacokinetics, and was associated with im-

Address correspondence to Dr. Reiner Frey, Clinical Pharmacology, Pharma Research Center, Bayer Pharma AG, Aprather Weg 18a, 42113 Wuppertal, Germany. E-mail: reiner.frey@bayer.com.

Submitted November 27, 2014; Accepted June 18, 2015; Electronically published March 11, 2016

C) 2016 by the Pulmonary Vascular Research Institute. All rights reserved. 2045-8932/2016/06S1-0002. \$15.00. 
provements in cardiac function and exercise capacity in patients with $\mathrm{PH}^{8-11}$ Riociguat is approved in more than 40 countries, including the United States and countries of the European Union.

Riociguat is metabolized to M1 (BAY 60-4552) via the cytochrome P450 (CYP) monooxygenases, predominantly CYP1A1 but also CYP3A4, CYP2C8, and CYP2J2. ${ }^{12}$ Low levels of CYP1A1 are expressed continuously; ${ }^{13}$ however, expression is induced by the polycyclic aromatic hydrocarbons found in tobacco smoke. ${ }^{14,15}$ Consequently, smokers are likely to have increased metabolic clearance of riociguat to M1. In healthy individuals, riociguat is excreted via both renal (approximately 30\%-45\%) and biliary/fecal (approximately $45 \%-60 \%$ ) routes. ${ }^{12,16}$ Given the substantial role played by the liver in the clearance of riociguat, it is important that the potential impact of hepatic impairment on the pharmacokinetics of riociguat be determined. The primary aim of this clinical pharmacology analysis was to investigate the pharmacokinetics and safety of riociguat following a single oral dose in individuals with mild or moderate hepatic impairment associated with liver cirrhosis, classified as Child-Pugh A or B, respectively, ${ }^{17,18}$ compared with that in age-, weight-, and sex-matched healthy controls. In addition, the effects of smoking on riociguat pharmacokinetics were evaluated.

\section{METHODS}

\section{Study design and treatments}

Two nonrandomized, nonblinded studies with group stratification were conducted at a single center in Germany (study 1, from November 2008 to June 2009; study 2, from April 2010 to April 2011). Study 1 and study 2 had the same design. Participants in both studies, including those with hepatic impairment and healthy controls, received a single oral dose of riociguat $1.0 \mathrm{mg}$ (as two immediaterelease tablets of $0.5 \mathrm{mg}$ ). In individuals with hepatic impairment, the planned morning dose of concomitant medication was withheld until $4 \mathrm{~h}$ after the riociguat dose. All participants remained at the study center for 3 days. Participants underwent a final examination in the 2-week period following study drug administration.

After study 1 had been finalized, it became evident that riociguat was metabolized to M1 not only via the CYP monooxygenases CYP3A4, CYP2C8, and CYP2J2, as previously known, but also via CYP1A $1 .{ }^{12}$ As expression of this enzyme is induced by tobacco smoke, ${ }^{14,15}$ exclusion of smokers as a significant confounder was considered to allow a more accurate assessment of the influence of hepatic impairment on riociguat pharmacokinetics, and therefore a second study (study 2) was conducted in which nonsmokers only were recruited. The results from the two studies have been combined and are presented together.

Both studies were conducted in accordance with the Declaration of Helsinki and adhered to the International Conference on Harmonization Good Clinical Practice Guidelines and the German drug law (AMG). The study protocols were approved by the Ethics Committee of the Schleswig-Holstein Medical Council, Bad Segeberg, Germany, and both studies complied with the US Food and Drug Administration (FDA) and European Medicines Agency (EMA) guidelines for industry. ${ }^{19,20}$ All individuals provided written informed consent to participate in the studies.

\section{Study population}

Individuals aged 18-75 years with liver cirrhosis (confirmed by histopathology or ultrasound) and mild (Child-Pugh A) or moderate (Child-Pugh B) hepatic impairment (see Table 1 for classification $)^{17,18,21}$ were eligible to participate if their body mass index (BMI) was in the range 18-32 ( $\leq 34$ for those with moderate impairment, to allow for possible ascites) and if they had a resting heart rate of 45-100 bpm, a systolic blood pressure (SBP) of 100-160 $\mathrm{mmHg}$, a diastolic blood pressure (DBP) no higher than $95 \mathrm{mmHg}$, and a negative drug screen result. Healthy individuals with a BMI in the range of 18-32, a resting heart rate of 45-90 bpm, an SBP of 100$145 \mathrm{mmHg}$, a DBP no higher than $95 \mathrm{mmHg}$, and a negative drug screen result were selected as controls and were matched for age ( \pm 10 years), weight $( \pm 10 \mathrm{~kg})$, and sex to eligible participants with Child-Pugh A or B hepatic impairment (control group A and B, respectively). The main exclusion criteria relating to both study 1 and study 2 are detailed in Table $2 .^{22}$ Use of medicinal products other than riociguat was not permitted during the study without consulting the investigator. Any concomitant medication was documented.

Equal numbers of individuals were recruited to study 1 and study 2 and the same inclusion and exclusion criteria were used, with the exception that smoking was not permitted in study 2 (nonsmoking

Table 1. Child-Pugh scoring system

\begin{tabular}{lccc}
\hline Parameter & 1 point & 2 points & 3 points \\
\hline Serum albumin, g/L & $>35$ & $28-35$ & $<28$ \\
Ascites & None & Small quantity & Large quantity \\
Serum bilirubin, $\mu \mathrm{mol} / \mathrm{L}$ & $<34$ & $34-51$ & $>51$ \\
Hepatic encephalopathy & None & I/II & III/IV \\
Prothrombin time (Quick test value), $\%$ & $>60$ & $40-60$ & $<40$ \\
\hline
\end{tabular}

Note: The degree of hepatic impairment was classified as follows: Child-Pugh A, 5 or 6 points; Child-Pugh B, 7-9 points; Child-Pugh C, $10-15$ points. ${ }^{17,18}$

${ }^{a}$ Hepatic encephalopathy was assessed using the West Haven criteria for semiquantitative grading of mental state. ${ }^{21}$ 
Table 2. Main exclusion criteria applicable to both studies

Exclusion criteria applicable to all individuals

History of medical disorders or conditions that could impair an individual's ability to participate in or complete the study

Febrile illness in the week before the study

History of severe allergies and nonallergic drug reactions

Pathological changes in the electrocardiogram, such as a secondor third-degree atrioventricular block, prolongation of the QRS complex to more than $120 \mathrm{~ms}$, or prolongation of the QT/QTc interval to more than $450 \mathrm{~ms}$

Participation in another clinical study in the previous 3 months

Donation of more than $100 \mathrm{~mL}$ of blood in the preceding 4 weeks or $500 \mathrm{~mL}$ in the preceding 3 months

Pregnant women and women of childbearing age not using double-barrier contraception

Exclusion criteria applicable to patients with liver cirrhosis and hepatic impairment

Concurrent diagnosis of severe cerebrovascular or cardiac disorders, renal failure with a $\mathrm{CL}_{\mathrm{CR}}$ of $40 \mathrm{~mL} / \mathrm{min}$ or lower (according to the Cockcroft-Gault formula), ${ }^{22}$ grade III or IV hepatic encephalopathy, or failure of any organ system other than the liver

Primary and secondary biliary cirrhosis

Sclerosing cholangitis

Diagnosis of malignancy within the previous 5 years

Severe infection or any clinically significant illness in the 4 weeks before drug administration

Percutaneous transluminal coronary angioplasty or coronary artery bypass graft less than 6 months before riociguat administration

Concomitant thrombotic disorders

Platelet count less than $50 \times 10^{9} / \mathrm{L}$

Diabetes mellitus with a fasting blood glucose level higher than $220 \mathrm{mg} / \mathrm{dL}$ or glycosylated hemoglobin levels of more than $10 \%$

History of bleeding in the past 3 months

Ascites of more than $6 \mathrm{~L}$ (estimated by ultrasound)

Prothrombin time (Quick test) below 40\%

Alkaline phosphatase level at least three times the ULN

Elevated AST or ALT in conjunction with a GGT level at least three times the ULN (an isolated elevation of GGT $\geq 3$ times the ULN did not result in exclusion from the study)

Cholinesterase lower than $1,800 \mathrm{U} / \mathrm{L}$

Total bilirubin higher than $102 \mu \mathrm{mol} / \mathrm{L}$

Concomitant use of potent CYP3A4 inhibitors, phosphodiesterase 5 inhibitors, endothelin receptor antagonists, or prostacyclins or treatment with any medication (except those necessary for the treatment of liver disease or related complications)

Change in chronic medication less than 4 weeks before dosing

Note: ALT: alanine aminotransferase; AST: aspartate aminotransferase; $\mathrm{CL}_{\mathrm{CR}}$ : creatinine clearance; GGT: glutamyl transpeptidase; ULN: upper limit of normal. individuals should not have smoked for at least 3 months). Study participants were allowed a daily consumption of not more than $40 \mathrm{~g}$ ( 5 units) of alcohol, $1 \mathrm{~L}$ of xanthine-containing beverages, and 25 cigarettes (study 1 only).

\section{Safety and tolerability}

Safety and tolerability were evaluated by monitoring standard vital signs, hematology, urinalysis, and biochemical tests. Adverse events (AEs) were identified by questioning of participants and through spontaneous self-reporting. All AEs were classified according to their degree of severity (mild, moderate, or severe) and whether they were serious.

\section{Pharmacokinetic evaluation}

Plasma and urine samples for determination of the pharmacokinetics of riociguat and its metabolite, M1, were obtained before and up to 72 hours after riociguat administration. Samples were stored below $-15^{\circ} \mathrm{C}$ until analysis.

In both studies, total (bound and unbound) plasma and urinary riociguat and M1 concentrations were determined by highperformance liquid chromatography coupled with mass spectrometry, using $\left[{ }^{2} \mathrm{H}_{3}\right]$ riociguat and $\left[{ }^{2} \mathrm{H}_{3}\right] \mathrm{M} 1$ as the internal standards. In study 1 , the working ranges were $0.500-100 \mu \mathrm{g} / \mathrm{L}$ in plasma and $10.0-1,000 \mu \mathrm{g} / \mathrm{L}$ in urine. For plasma, quality control (QC) samples in the concentration range $1.50-80.0 \mu \mathrm{g} / \mathrm{L}$ were determined with an accuracy of $106 \%-108 \%$ and a precision of $4.21 \%-7.34 \%$ for riociguat and with an accuracy of $103 \%-107 \%$ and a precision of $4.05 \%-$ $6.86 \%$ for M1. For urine, QC samples in the concentration range $30.0-800 \mu \mathrm{g} / \mathrm{L}$ were determined with an accuracy of $101 \%-104 \%$ and a precision of $3.54 \%-3.71 \%$ for riociguat and with an accuracy of $97.8 \%-99.0 \%$ and a precision of $2.63 \%-5.41 \%$ for M1.

In study 2 , the working ranges were $0.500-250 \mu \mathrm{g} / \mathrm{L}$ in plasma and $10.0-1,000 \mu \mathrm{g} / \mathrm{L}$ in urine. For plasma, QC samples in the concentration range $1.50-200 \mu \mathrm{g} / \mathrm{L}$ were determined with an accuracy of $98.2 \%-101 \%$ and a precision of $4.45 \%-5.50 \%$ for riociguat and with an accuracy of $97.0 \%-99.0 \%$ and a precision of $4.49 \%-7.48 \%$ for M1. For urine, QC samples in the concentration range 30.0$800 \mu \mathrm{g} / \mathrm{L}$ were determined with an accuracy of $99.1 \%-103 \%$ and a precision of $4.88 \%-6.19 \%$ for riociguat and with an accuracy of $98.0 \%-104 \%$ and a precision of $2.53 \%-8.87 \%$ for M1.

In both studies, determination of protein binding was performed in vitro according to the method of Schuhmacher et al., ${ }^{23}$ using $\left[{ }^{14} \mathrm{C}\right]$-labeled riociguat and $\left[{ }^{14} \mathrm{C}\right]$-labeled M1 as the test substances.

The following pharmacokinetic characteristics were calculated for total (bound and unbound) riociguat and M1 using noncompartmental methods in WinNonlin (ver. 4.1a; Pharsight, Mountain View, CA): area under the plasma concentration-time curve from time 0 to infinity (AUC), AUC divided by dose of riociguat per kilogram of body weight $\left(\mathrm{AUC}_{\text {norm }}\right)$, maximum concentration in plasma $\left(C_{\max }\right), C_{\max }$ divided by dose of riociguat per kilogram of body weight ( $\left.C_{\max , \text { norm }}\right)$, time to $C_{\max }\left(t_{\max }\right)$, terminal elimination half-life $\left(t_{1 / 2}\right)$, apparent oral clearance $(\mathrm{CL} / F)$, amount excreted into urine from time 0 to infinity $\left(A_{\mathrm{E}, \mathrm{ur}}\right)$, and renal clearance $\left(\mathrm{CL}_{\mathrm{R}}\right)$. Corresponding parameters for unbound riociguat and $\mathrm{M} 1\left(\mathrm{AUC}_{\mathrm{u}}, \mathrm{AUC}_{\mathrm{u}, \mathrm{norm}}, C_{\mathrm{max}, \mathrm{u}}\right.$, 
$\left.C_{\text {max,unorm }}\right)$ were calculated using the fraction unbound $\left(f_{\mathrm{u}}\right)$. The linear-logarithmic trapezoidal method was used to calculate AUC; $t_{1 / 2}$ was calculated by linear least-squares (LS) regression after logarithmic transformation of the (three or more) terminal concentrations.

\section{Statistical analysis}

Statistical evaluation was performed using SAS software (ver. 9.1 and 9.2; SAS Institute, Cary, NC). An integrated analysis of the two studies was performed to evaluate the effect of hepatic impairment on the pharmacokinetics of riociguat and the metabolite M1 in a mixed population of smokers and nonsmokers with an enrichment of nonsmokers, considered to be similar to the target population.

The pharmacokinetic characteristics $\mathrm{AUC}, \mathrm{AUC} \mathrm{C}_{\text {norm }}, C_{\max }$, and $C_{\text {max,norm }}$ of riociguat and $\mathrm{M} 1$ were analyzed assuming that the data followed a lognormal distribution. To compare the pharmacokinetics between groups of different hepatic function, the logarithms of these pharmacokinetic characteristics were assessed using analysis of variance (ANOVA), including a group effect. On the basis of these results, point estimates (LS means) and exploratory 90\% confidence intervals (CIs) for the ratios Child-Pugh A/control A and ChildPugh $\mathrm{B} /$ control $\mathrm{B}$ were calculated by retransformation of the logarithmic data derived from the ANOVA. Further explorative analyses, including a graphical description of the relationship between hepatic function and selected pharmacokinetic parameters, were carried out.

A subtotal of 16 participants per group was considered sufficient to achieve the objectives of the investigation. Assuming that the coefficient of variation observed with primary pharmacokinetic targets reached $75 \%$ and a true mean ratio of 2 , an increase in the primary pharmacokinetic parameters could be confirmed with a power of $83 \%$ on the basis of a sample size of at least 14 matched pairs (SAS Proc Power; one-sided paired $t$ test of mean ratio with lognormal data; $\alpha=0.05)$

\section{RESULTS}

\section{Study population}

In total, 64 individuals (52 nonsmokers and 12 smokers; 42 men and 22 women; mean age: 55.1 years; age range: $35-72$ years) re- ceived riociguat and provided valid data for the safety evaluation and pharmacokinetic analyses (Table 3 ). The mean \pm standard deviation weight was $82.6 \pm 13.9 \mathrm{~kg}$, mean height was $173.7 \pm 8.9 \mathrm{~cm}$, and mean BMI was $27.2 \pm 3.2$. In each study, there were eight patients with Child-Pugh A hepatic impairment, eight patients with Child-Pugh B hepatic impairment, and 16 controls.

\section{Safety and tolerability}

No serious AEs occurred during these studies. Of 64 participants, 21 (33\%) experienced at least one treatment-emergent AE following riociguat administration. Twelve (19\%) participants experienced at least one $\mathrm{AE}$ assessed by the investigator as being drug related. Headache was the most frequently reported drug-related AE, occurring in $6(19 \%)$ healthy controls and in 4 (13\%) individuals in the Child-Pugh A group. There was no suggestion of an increase in the incidence of drug-related AEs due to hepatic impairment. There was also no indication that the study drug was associated with any clinically relevant changes to laboratory parameters.

\section{Pharmacokinetics}

The pharmacokinetic parameters of riociguat and M1 in both individuals with hepatic impairment and matched healthy controls are summarized in Table 4. Owing to the rapid absorption of riociguat (median $t_{\max }$ values ranged between 0.750 hours among individuals with Child-Pugh A hepatic impairment and 1.50 hours among individuals in control group $\mathrm{A}$ ), the mean $C_{\max \text {,norm }}$ of total riociguat was similar in all four groups (Table 4; Fig. $1 A$ ). The mean $t_{1 / 2}$ of total riociguat was prolonged in individuals with Child-Pugh $\mathrm{B}$ hepatic impairment (13.7 hours; geometric mean) compared with that in individuals with Child-Pugh A impairment (9.19 hours) and matched healthy controls (9.02 and 7.54 hours for control groups A and B, respectively; Table 4).

Exposure (AUC) to riociguat was elevated among individuals with Child-Pugh B hepatic impairment, but not among those with Child-Pugh A hepatic impairment, compared with that in the respective groups of healthy controls. In individuals with ChildPugh B hepatic impairment, the mean exposure to total riociguat

Table 3. Baseline demographic characteristics of healthy individuals (normal hepatic function; controls) and patients with mild (ChildPugh A) or moderate (Child-Pugh B) hepatic impairment who were valid for pharmacokinetic analysis (total population of smokers and nonsmokers)

\begin{tabular}{lccccc}
\hline Demographic characteristic & $\begin{array}{c}\text { Child-Pugh A } \\
(n=16)\end{array}$ & $\begin{array}{c}\text { Child-Pugh B } \\
(n=16)\end{array}$ & $\begin{array}{c}\text { Control A } \\
(n=16)\end{array}$ & $\begin{array}{c}\text { Control B } \\
(n=16)\end{array}$ & $\begin{array}{c}\text { Total } \\
(N=64)\end{array}$ \\
\hline Age, ${ }^{\text {a }}$ years & $55.3(42.0-72.0)$ & $56.7(39.0-70.0)$ & $52.3(35.0-69.0)$ & $56.1(37.0-67.0)$ & $55.1(35.0-72.0)$ \\
Female, no. (\%) & $5(31)$ & $6(38)$ & $5(31)$ & $6(38)$ & $22(34)$ \\
White, no. (\%) & $16(100)$ & $16(100)$ & $16(100)$ & $16(100)$ & $64(100)$ \\
BMI $^{\text {b }}$ & $27.2(4.0)$ & $26.8(3.4)$ & $27.6(3.1)$ & $27.3(2.1)$ & $27.2(3.2)$ \\
CL $_{\text {CR }}$, mL/min & $90.1(23.0)$ & $79.0(20.0)$ & $94.2(19.9)$ & $85.8(17.1)$ & Not calculated \\
Current smoker, no. (\%) & $3(19)$ & $5(31)$ & $2(13)$ & $2(13)$ & $12(19)$ \\
\hline
\end{tabular}

Note: BMI: body mass index; $\mathrm{CL}_{\mathrm{CR}}$ : creatinine clearance.

${ }^{\text {a }}$ Arithmetic mean (range).

${ }^{\mathrm{b}}$ Arithmetic mean (standard deviation). 
Table 4. Pharmacokinetic parameters for riociguat and metabolite M1 in healthy individuals (controls) and patients with mild (ChildPugh A) or moderate (Child-Pugh B) hepatic impairment, following a single oral dose of riociguat $1.0 \mathrm{mg}$ (total population of smokers and nonsmokers)

\begin{tabular}{|c|c|c|c|c|}
\hline Parameter & Child-Pugh A $(n=16)$ & Child-Pugh B $(n=16)$ & Control A $(n=16)$ & Control B $(n=16)$ \\
\hline \multicolumn{5}{|l|}{ Riociguat } \\
\hline AUC, $\mu \mathrm{g} \cdot \mathrm{h} / \mathrm{L}$ & $371(74)$ & $459(62)$ & $350(67)$ & $301(92)$ \\
\hline $\mathrm{AUC}_{\text {norm }}, \mathrm{kg} \cdot \mathrm{h} / \mathrm{L}$ & $30.9(75)$ & $36.6(65)$ & $29.1(67)$ & $23.9(94)$ \\
\hline$C_{\max }, \mu \mathrm{g} / \mathrm{L}$ & $42.7(37)$ & $43.3(39)$ & $42.7(23)$ & $38.7(30)$ \\
\hline$C_{\text {max,norm }}, \mathrm{kg} / \mathrm{L}$ & $3.56(33)$ & $3.45(26)$ & $3.55(20)$ & $3.07(23)$ \\
\hline$t_{\max }$, hours & $0.750(0.500-3.00)$ & $1.25(0.750-4.00)$ & $0.875(0.500-3.00)$ & $1.50(0.500-3.00)$ \\
\hline$t_{1 / 2}$, hours & $9.19(53)$ & $13.7(50)$ & $9.02(63)$ & $7.54(86)$ \\
\hline $\mathrm{CL} / F, \mathrm{~L} / \mathrm{h}$ & $2.70(74)$ & $2.18(62)$ & $2.86(67)$ & $3.32(92)$ \\
\hline$f_{\mathrm{u}}, \%$ & $3.23(22)$ & $3.81(37)$ & $3.34(20)$ & $3.45(23)$ \\
\hline $\mathrm{AUC}_{\mathrm{u}, \text { norm }}, \mathrm{kg} \cdot \mathrm{h} / \mathrm{L}$ & $0.999(82)$ & $1.39(92)$ & $0.972(65)$ & $0.825(93)$ \\
\hline$C_{\max , \mathrm{u}, \text { norm }}, \mathrm{kg} / \mathrm{L}$ & $0.115(35)$ & $0.131(35)$ & $0.119(23)$ & $0.106(25)$ \\
\hline$A_{\mathrm{E}, \mathrm{ur}}, \%$ & $9.52 \pm 4.79$ & $12.2 \pm 8.68$ & $11.4 \pm 5.26$ & $9.69 \pm 4.69$ \\
\hline $\mathrm{CL}_{\mathrm{R}}, \mathrm{L} / \mathrm{h}$ & $0.226(62)$ & $0.226(85)$ & $0.289(30)$ & $0.280(43)$ \\
\hline \multicolumn{5}{|l|}{ Metabolite M1 } \\
\hline AUC, $\mu \mathrm{g} \cdot \mathrm{h} / \mathrm{L}$ & $289(52)$ & $266(56)$ & $213(38)$ & $222(35)$ \\
\hline $\mathrm{AUC}_{\text {norm }}, \mathrm{kg} \cdot \mathrm{h} / \mathrm{L}$ & $24.9(44)$ & $21.9(60)$ & $18.4(34)$ & $18.2(31)$ \\
\hline$C_{\max }, \mu \mathrm{g} / \mathrm{L}$ & $8.41(78)$ & $6.55(79)$ & $6.86(68)$ & $7.79(73)$ \\
\hline$C_{\max , \text { norm }}, \mathrm{kg} / \mathrm{L}$ & $0.725(70)$ & $0.540(69)$ & $0.591(64)$ & $0.640(66)$ \\
\hline$t_{\max }$, hours & $12.0(2.00-48.0)$ & $12.0(2.00-48.0)$ & $8.00(2.00-24.0)$ & $12.0(3.00-24.0)$ \\
\hline$t_{1 / 2}$, hours & $19.0(32)$ & $22.2(49)$ & $15.3(32)$ & $15.5(36)$ \\
\hline $\mathrm{CL} / F, \mathrm{~L} / \mathrm{h}$ & $3.34(52)$ & $3.64(56)$ & $4.53(38)$ & $4.36(35)$ \\
\hline$f_{\mathrm{u}}, \%$ & $2.81(25)$ & $3.57(32)$ & $2.91(20)$ & $3.01(20)$ \\
\hline $\mathrm{AUC}_{\mathrm{u}, \text { norm }}, \mathrm{kg} \cdot \mathrm{h} / \mathrm{L}$ & $0.702(53)$ & $0.787(55)$ & $0.535(47)$ & $0.548(38)$ \\
\hline$C_{\text {max,u,norm }}, \mathrm{kg} / \mathrm{L}$ & $0.0204(76)$ & $0.0193(50)$ & $0.0172(72)$ & $0.0193(70)$ \\
\hline$A_{\mathrm{E}, \mathrm{ur}}, \%$ & $13.2 \pm 7.42$ & $7.29 \pm 4.57^{\mathrm{a}}$ & $15.2 \pm 8.57$ & $14.1 \pm 6.68$ \\
\hline $\mathrm{CL}_{\mathrm{R}}, \mathrm{L} / \mathrm{h}$ & $0.396(88)$ & $0.234(98)^{\mathrm{a}}$ & $0.618(49)$ & $0.560(59)$ \\
\hline
\end{tabular}

Note: Data are geometric means (percentage coefficient of variation) except for $t_{\max }$, which is expressed as median (range), and $A_{\mathrm{E}, \mathrm{ur}}$, which is expressed as arithmetic mean \pm standard deviation. $A_{\mathrm{E}, \mathrm{ur}}$ : amount excreted into urine from time 0 to infinity; AUC: area under the plasma concentration-time curve from time 0 to infinity; $\mathrm{AUC}_{\text {norm: }} \mathrm{AUC}$ divided by dose of riociguat per kilogram of body weight for total (bound and unbound) riociguat/M1; $\mathrm{AUC}_{\mathrm{u}, \mathrm{norm}}$ : $\mathrm{AUC}$ divided by dose of riociguat per kilogram of body weight for unbound riociguat/M1; CL/F: apparent oral clearance for total riociguat/M1; $\mathrm{CL}_{\mathrm{R}}$ : renal clearance of riociguat/M1; $C_{\max }$ : maximum concentration in plasma; $C_{\max , \text { norm }}$ : $C_{\max }$ divided by dose of riociguat per kilogram of body weight for total riociguat/M1; $C_{\max , \mathrm{u} \text {,norm }}: C_{\max }$ divided by dose of riociguat per kilogram of body weight for unbound riociguat/M1; $f_{\mathrm{u}}$ : fraction unbound; $t_{\max }$ : time to $C_{\max }$ of total riociguat/M1; $t_{1 / 2}$ : terminal elimination half-life for total riociguat/M1.

${ }^{a}$ Data were available from 15 patients.

$\left(\mathrm{AUC}_{\text {norm }}\right)$ was increased by approximately $50 \%$ compared with that in matched healthy controls, and the mean exposure to unbound riociguat $\left(\mathrm{AUC}_{\mathrm{u}, \text { norm }}\right)$ was increased by almost $70 \%$ (Table 4$)$. The lower limits of the $90 \%$ CIs for the ratio Child-Pugh B/control B for total $\left(\mathrm{AUC}_{\mathrm{norm}}\right)$ and unbound $\left(\mathrm{AUC}_{\mathrm{u}, \mathrm{norm}}\right)$ riociguat in plasma were greater than $100 \%$, indicating that the corresponding increases reached statistical significance (Table 5). The $f_{\mathrm{u}}$ of riociguat in individuals with Child-Pugh B hepatic impairment was increased by approximately $10 \%$ compared with that observed among healthy individuals in the control groups (Table 4). In terms of mean $\mathrm{AUC}_{\text {norm }}$ values of total and unbound riociguat, exposure was similar in indi- viduals with Child-Pugh A hepatic impairment and in matched healthy controls (Table 4). In general, a rank order of exposure in terms of $\mathrm{AUC}_{\text {norm }}$ (individuals with Child-Pugh B hepatic impairment $>$ individuals with Child-Pugh A hepatic impairment $\geq$ healthy controls) was observed in the pooled analysis (Table 4; Fig. 2A).

Individuals with hepatic impairment had a reduced rate of formation and impaired elimination of M1 compared with healthy controls (Table 4 ). The $t_{1 / 2}$ of M1 was prolonged by approximately $43 \%$ among individuals with Child-Pugh B hepatic impairment and by approximately $24 \%$ among individuals with Child-Pugh A hepatic impairment compared with their respective matched controls (Ta- 

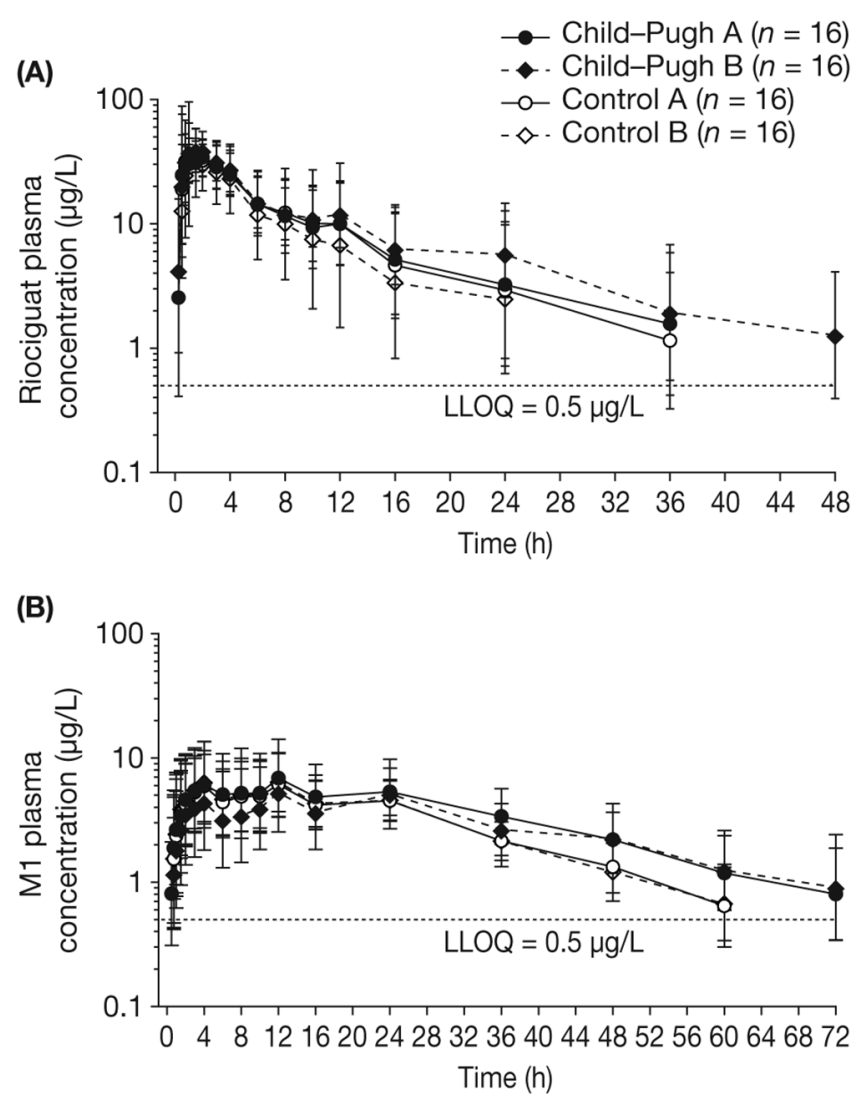

Figure 1. Geometric mean plasma concentration-time courses of total riociguat $(A)$ and metabolite $\mathrm{M} 1(B)$ after a single oral dose of riociguat $1.0 \mathrm{mg}$ in eligible participants with Child-Pugh A or B hepatic impairment or the respective age-, weight-, and sex-matched healthy controls. Bars denote the geometric standard deviation; semilogarithmic scale; $N=64$ (all individuals eligible for pharmacokinetic evaluation; total population of smokers and nonsmokers). Individual data values below the lower limit of quantification (LLOQ) ceased to be measured. Mean values at any one time were calculated only if at least two-thirds of individual data were above the LLOQ. Values below the LLOQ were substituted by half of this limit for calculation of mean values.

ble 4). The median $t_{\max }$ for M1 ranged between 8.00 hours (control group A) and 12.0 hours (both groups of hepatic impairment and control group B; Table 4; Fig. 1B). The antagonizing effects of diminished M1 formation and elimination led to only small differences in overall exposure ( $\mathrm{AUC}_{\text {norm }}$ ) to $\mathrm{M} 1$ between individuals with Child-Pugh A (24.9 kg.h/L) or Child-Pugh B $(21.9 \mathrm{~kg} \cdot \mathrm{h} / \mathrm{L})$ hepatic impairment and their matched healthy controls (18.4 and $18.2 \mathrm{~kg} \cdot \mathrm{h} / \mathrm{L}$, respectively; Table 4; Fig. 2B; Fig. 3B). The ratios (90\% CIs) of LS means of $C_{\max \text {,norm }}$ between patients with hepatic impairment and matched healthy individuals for total M1 were 84.4 (58.9-121) for Child-Pugh B and 123 (85.6-176) for Child-Pugh A (Table 5). There was little variation in the $C_{\max , \mathrm{u}, \text { norm }}$ of M1 between the hepatic-function groups (Table 4).

Although the total levels of M1 were similar in the whole study population (comprising nonsmokers and smokers; Fig. $2 B$ ) and in the population of nonsmokers only (Fig. $3 B$ ), individuals who did not smoke had a higher exposure to riociguat per se (median $\mathrm{AUC}_{\text {norm }}$ of nonsmokers vs. whole population: Child-Pugh A, 43.3 vs. $32.7 \mathrm{~kg} \cdot \mathrm{h} / \mathrm{L}$; Child-Pugh B, 39.4 vs. $36.3 \mathrm{~kg} \cdot \mathrm{h} / \mathrm{L}$; Fig. 2 A, $3 A$ ), most

Table 5. Point estimates (least-squares means) and two-sided $90 \%$ confidence intervals (CIs) of selected pharmacokinetic parameters for total and unbound riociguat and metabolite M1 in plasma from patients (total population of smokers and nonsmokers) with mild (Child-Pugh A) or moderate (Child-Pugh B) hepatic impairment compared with those in the respective groups of healthy individuals (controls), following a single oral dose of riociguat $1.0 \mathrm{mg}$

\begin{tabular}{|c|c|c|c|c|}
\hline Ratio, parameter & $n$ & $\begin{array}{c}\mathrm{CV}, \\
\%\end{array}$ & $\begin{array}{c}\text { Estimated } \\
\text { ratio, \% }\end{array}$ & $\begin{array}{c}90 \% \\
\text { CI, \% }\end{array}$ \\
\hline \multicolumn{5}{|c|}{ Riociguat (total) } \\
\hline \multicolumn{5}{|c|}{ Child-Pugh A/control A } \\
\hline $\mathrm{AUC}_{\text {norm }}$ & 32 & 75 & 106 & $71.4-158$ \\
\hline$C_{\max , \text { norm }}$ & 32 & 26 & 100 & $86.1-116$ \\
\hline \multicolumn{5}{|c|}{ Child-Pugh B/control B } \\
\hline $\mathrm{AUC}_{\text {norm }}$ & 32 & 75 & 153 & $103-228$ \\
\hline$C_{\text {max,norm }}$ & 32 & 26 & 112 & $96.7-130$ \\
\hline \multicolumn{5}{|c|}{ Metabolite M1 (total) } \\
\hline \multicolumn{5}{|c|}{ Child-Pugh A/control A } \\
\hline $\mathrm{AUC}_{\text {norm }}$ & 32 & 43 & 136 & $106-174$ \\
\hline$C_{\text {max,norm }}$ & 32 & 67 & 123 & $85.6-176$ \\
\hline \multicolumn{5}{|c|}{ Child-Pugh B/control B } \\
\hline $\mathrm{AUC}_{\text {norm }}$ & 32 & 43 & 121 & $94.2-154$ \\
\hline$C_{\text {max,norm }}$ & 32 & 67 & 84.4 & $58.9-121$ \\
\hline \multicolumn{5}{|c|}{ Riociguat (unbound) } \\
\hline \multicolumn{5}{|c|}{ Child-Pugh A/control A } \\
\hline $\mathrm{AUC}_{\mathrm{u}, \text { norm }}$ & 32 & 83 & 103 & $66.9-158$ \\
\hline$C_{\text {max,u,norm }}$ & 32 & 30 & 96.9 & $81.5-115$ \\
\hline \multicolumn{5}{|c|}{ Child-Pugh B/control B } \\
\hline $\mathrm{AUC}_{\mathrm{u}, \mathrm{norm}}$ & 32 & 83 & 169 & $110-259$ \\
\hline$C_{\max , \mathrm{u}, \mathrm{norm}}$ & 32 & 30 & 124 & $104-147$ \\
\hline \multicolumn{5}{|c|}{ Metabolite M1(unbound) } \\
\hline \multicolumn{5}{|c|}{ Child-Pugh A/control A } \\
\hline $\mathrm{AUC}_{\mathrm{u}, \mathrm{norm}}$ & 32 & 48 & 131 & $100-172$ \\
\hline$C_{\text {max,u,norm }}$ & 32 & 67 & 119 & $82.7-170$ \\
\hline \multicolumn{5}{|c|}{ Child-Pugh B/control B } \\
\hline $\mathrm{AUC}_{\mathrm{u}, \text { norm }}$ & 32 & 48 & 143 & $109-188$ \\
\hline$C_{\text {max,u,norm }}$ & 32 & 67 & 100 & $69.9-144$ \\
\hline
\end{tabular}

Note: $\mathrm{AUC}_{\text {norm }}$ : area under the plasma concentration-time curve from time 0 to infinity divided by dose of riociguat per kilogram of body weight for total riociguat/M1; $\mathrm{AUC}_{\mathrm{u}, \text { norm: }}$ area under the plasma concentration-time curve from time 0 to infinity divided by dose of riociguat per kilogram of body weight for unbound riociguat/M1; $C_{\text {max,norm: }}$ maximum concentration in plasma divided by dose of riociguat per kilogram of body weight for total riociguat/M1; $C_{\max , u, n o r m:}$ maximum concentration in plasma divided by dose of riociguat per kilogram of body weight for unbound riociguat/M1; CV: coefficient of variation. 
(A)

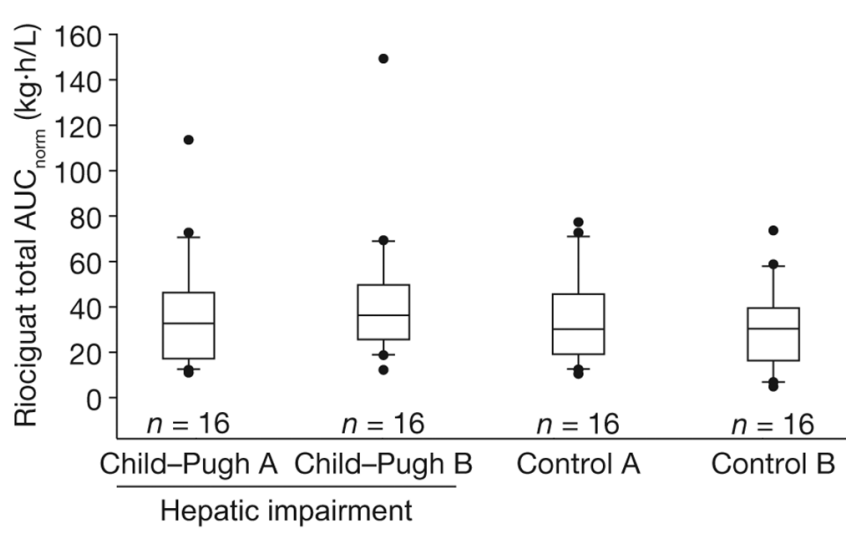

(B)

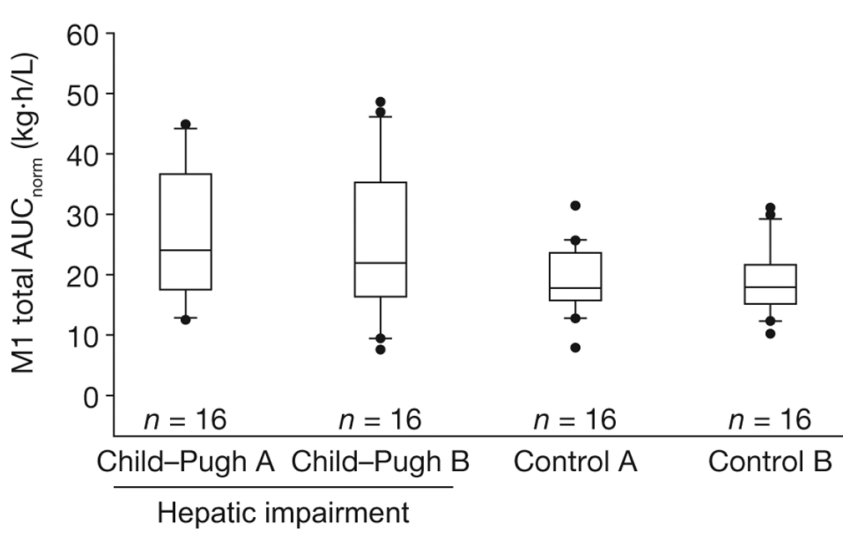

Figure 2. Box-and-whisker plots of exposure to total riociguat $(A)$ and metabolite M1 $(B)$, normalized for riociguat dose and body weight $\left(\mathrm{AUC}_{\text {norm }}\right)$, following a single oral dose of riociguat $1.0 \mathrm{mg}$ in a mixed population of smokers and nonsmokers $(N=64$, all individuals eligible for pharmacokinetic evaluation). Study groups consisted of eligible participants with Child-Pugh A or B hepatic impairment and respective age-, weight-, and sex-matched healthy controls. Boxes signify the 25th to 75 th percentiles, vertical lines show the 10th to 90th percentiles, and horizontal bisecting lines represent medians. More extreme values are plotted as points. $\mathrm{AUC}_{\text {norm }}$, area under the plasma concentration-time curve from time 0 to infinity divided by dose of riociguat per kilogram of body weight for total riociguat.

probably as a result of a lower metabolic clearance of riociguat to M1 compared with individuals who smoked. Thus, impaired hepatic function resulted in a higher mean exposure to riociguat in nonsmokers than was seen in the respective groups of impaired hepatic function of the total population (Tables $4, \mathrm{~S} 1$, available online).

The mean $\mathrm{CL}_{\mathrm{R}}$ of riociguat was lower in individuals with ChildPugh A or B hepatic impairment than in their matched healthy controls (Table 4). $\mathrm{CL}_{\mathrm{CR}}$ values measured on the treatment day indicated decreased renal function (in addition to hepatic impairment) in individuals with Child-Pugh A $\left(\mathrm{CL}_{\mathrm{CR}}\right.$ of $\left.94.2 \pm 31.1 \mathrm{~mL} / \mathrm{min}\right)$ or Child-Pugh $\mathrm{B}\left(\mathrm{CL}_{\mathrm{CR}}\right.$ of $\left.78.6 \pm 29.5 \mathrm{~mL} / \mathrm{min}\right)$ hepatic impairment, whereas healthy individuals exhibited normal $\mathrm{CL}_{\mathrm{CR}}$ levels $\left(\mathrm{CL}_{\mathrm{CR}}\right.$ of
$121 \pm 48.1$ and $103 \pm 37.2 \mathrm{~mL} / \mathrm{min}$ for control groups $\mathrm{A}$ and $\mathrm{B}$, respectively). The effect of renal impairment is reflected by the reduced $A_{\mathrm{E}, \text { ur }}$ values for M1 observed in individuals with Child-Pugh A and, in particular, Child-Pugh B hepatic impairment compared with the $A_{\mathrm{E} \text {,ur }}$ values observed in individuals in the respective control groups (Table 4).

\section{DISCUSSION}

The liver plays a substantial role in the metabolism and clearance of riociguat. These two clinical pharmacological studies and their joint evaluation investigated the pharmacokinetics and safety of a single oral dose of riociguat $1.0 \mathrm{mg}$ in patients with mild or moderate hepatic impairment.

\section{(A)}

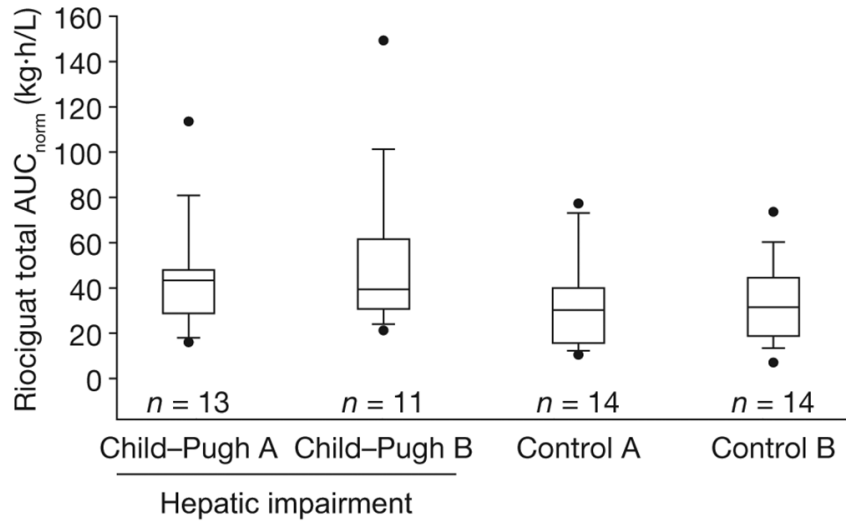

(B)

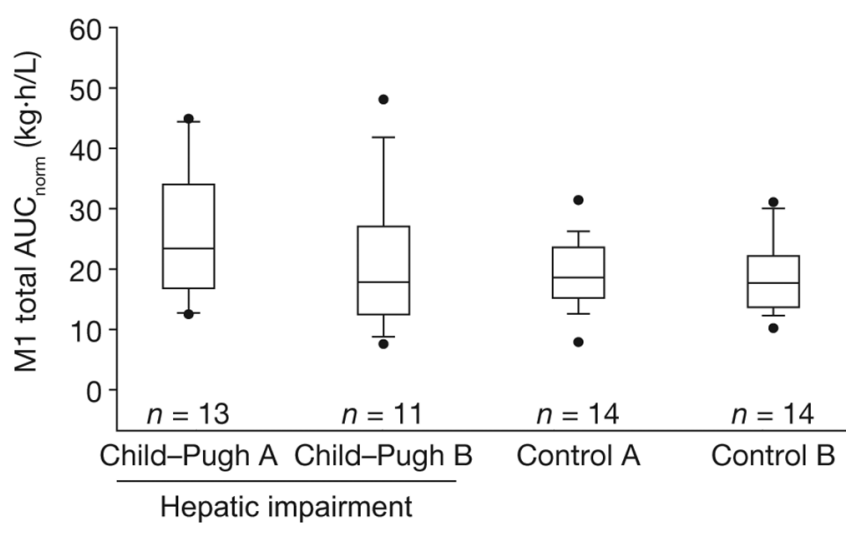

Figure 3. Box-and-whisker plots of exposure to total riociguat $(A)$ and metabolite M1 $(B)$, normalized for riociguat dose and body weight $\left(\mathrm{AUC}_{\text {norm }}\right)$, following a single oral dose of riociguat $1.0 \mathrm{mg}$ in a population of nonsmokers only $(N=52)$. Study groups consisted of eligible participants with Child-Pugh A or B hepatic impairment and respective age-, weight-, and sex-matched healthy controls. Boxes signify the 25th to 75 th percentiles, vertical lines show the 10th to 90th percentiles, and horizontal bisecting lines represent medians. More extreme values are plotted as points. $\mathrm{AUC}_{\text {norm }}$, area under the plasma concentration-time curve from time 0 to infinity divided by dose of riociguat per kilogram of body weight for total riociguat. 
Riociguat was well tolerated and showed a favorable safety profile. Exposure to riociguat was highly variable among individuals with hepatic impairment, and the ranges of AUC and $C_{\max }$ in patients with hepatic impairment overlapped with those previously observed in healthy volunteers and patients with $\mathrm{PH}$ receiving a single dose of riociguat $1.0 \mathrm{mg}$ (in solution). ${ }^{8,9} \mathrm{In}$ our group of smokers and nonsmokers with Child-Pugh A hepatic impairment, exposure to the drug was similar to that seen in matched healthy individuals. However, among patients with Child-Pugh B hepatic impairment, exposure to riociguat was increased by approximately $50 \%$ compared with that in matched healthy individuals, reflecting the reduced ability of patients with hepatic insufficiency to metabolize the drug to M1. Nonsmokers with Child-Pugh A or B hepatic impairment experience higher exposures to riociguat than the respective hepatic-impairment groups in the total population of smokers and nonsmokers, as described in "Results" and demonstrated in Figures 2 and 3. Consequently, the EMA and FDA labels for riociguat $^{12,24}$ are based on data relating to nonsmoking individuals, who might be at a higher risk of hypotension with riociguat than smokers owing to increased exposure; a statement on riociguat exposures in nonsmoking individuals with hepatic impairment is included in the EMA label as follows: "In cirrhotic patients (nonsmokers) with mild hepatic impairment (classified as Child Pugh A) riociguat mean AUC was increased by $35 \%$ compared to healthy controls, which is within normal intra-individual variability. In cirrhotic patients (nonsmokers) with moderate hepatic impairment (classified as Child Pugh B), riociguat mean AUC was increased by $51 \%$ compared to healthy controls. There are no data in patients with severe hepatic impairment (classified as Child Pugh C)." ${ }^{24}$

Overall, the increases in exposures to riociguat in patients with hepatic impairment were less than expected given the results of a mass balance study conducted in healthy volunteers, in which between $48 \%$ and $59 \%$ of riociguat-associated radioactivity from a single 1.0-mg oral dose of $\left[{ }^{14} \mathrm{C}\right]$ riociguat was eliminated via the biliary/ fecal route. ${ }^{25}$ Systemic clearance thus has an important role to play alongside hepatic clearance in the elimination of riociguat. These exposure data suggest that particular care should be exercised during individual dose titration for patients with Child-Pugh B hepatic impairment but that no additional precautions are necessary during dose titration for patients with mild (Child-Pugh A) hepatic impairment. This is reflected in the EMA label and is important because hepatic cirrhosis might be indolent and asymptomatic and therefore remain undetected until decompensated complications of hepatic disease present. ${ }^{26}$ Despite the differing levels of exposure, the safety profile of riociguat in individuals with hepatic impairment was similar to that in healthy individuals.

The effect of smoking on riociguat exposure that was documented in this study may be related to the sensitivity of the CYP1A1 protein to components of tobacco smoke. In humans, the microsomal enzyme CYP1A1 has been shown to be expressed in the lungs $s^{27-29}$ in addition to the liver ${ }^{30,31}$ and other extrahepatic tissues. ${ }^{31}$ CYP1A1 gene expression occurs at very low levels in uninduced cells; ${ }^{32}$ the messenger RNA is typically undetectable in the lungs of nonsmokers but is found at high levels in the bronchial epithelial cells of smokers. Likewise, the CYP1A1 protein is expressed at constitutively low levels in the lung tissues of nonsmokers but is found at much higher levels in the lung microsomes of smokers. ${ }^{13,33}$ Furthermore, interindividual variation in levels of CYP1A1 expression in lung samples is partially dependent on the number of cigarettes smoked per day. ${ }^{29}$ This small study suggests that, most probably owing to the enhanced expression of CYP1A1 induced by the polycyclic aromatic hydrocarbons found in tobacco smoke, ${ }^{14,15}$ the metabolism of riociguat to $\mathrm{M} 1$ is enhanced in a population including smokers, resulting in an overall reduced exposure to riociguat compared with that in a population of only nonsmokers. It would be of interest to discover in the future whether larger studies, perhaps based on registry data, can provide further insight into the relationship among hepatic function, smoking, and riociguat metabolism.

Mean $\mathrm{CL}_{\mathrm{CR}}$ at baseline ranged from $79.0 \mathrm{~mL} / \mathrm{min}$ in individuals with moderate hepatic impairment to $94.2 \mathrm{~mL} / \mathrm{min}$ in control group A. Following exposure to riociguat, mean $\mathrm{CL}_{\mathrm{CR}}$ and $\mathrm{CL}_{\mathrm{R}}$ of riociguat observed in patients in the Child-Pugh A and Child-Pugh B groups was lower than that in healthy controls. However, results from another recent clinical trial, which investigated the pharmacokinetics of riociguat in patients with mild, moderate, or severe renal impairment $\left(\mathrm{CL}_{\mathrm{CR}}\right.$ of $\left.15-80 \mathrm{~mL} / \mathrm{min}\right)$, suggest that there are no additional risks from the use of riociguat in these individuals; a greater overall exposure to riociguat was observed in patients with renal impairment $(42.7 \%-104.3 \%)$ than in healthy individuals, but the ranges of exposure in the study groups overlapped. ${ }^{34}$

Riociguat is approved for the treatment of patients with $\mathrm{PAH}$ and chronic thromboembolic PH (CTEPH). However, this study did not include patients with either form of $\mathrm{PH}$, a limitation that should be considered when extrapolating the findings into clinical practice. The absorption of riociguat (at therapeutic doses titrated up to $2.5 \mathrm{mg}$ three times daily) is similar in patients with $\mathrm{PH}$ and healthy individuals; however, riociguat elimination is markedly lower in patients with $\mathrm{PH}$, resulting in an approximately two- to threefold higher exposure compared with healthy individuals. ${ }^{35}$ Characteristics of patients with $\mathrm{PH}$, such as right-sided heart failure and increased age, are associated with physiological changes including diminished renal excretion and hepatobiliary elimination. The effect of underlying $\mathrm{PH}$ with right-sided heart failure on the organs responsible for drug elimination is hypothesized to influence riociguat exposure to a great extent. Right-sided heart failure is often caused by severe PAH and can result in hepatic dysfunction. The primary pathophysiology of this frequently asymptomatic condition is either hepatic congestion or impaired perfusion, the latter being associated with hepatic necrosis and reduced enzyme activity. ${ }^{36}$ In addition, the increased central venous pressures characteristic of $\mathrm{PH}$ with right heart failure are associated with worsening renal function. ${ }^{37}$ Despite the increased exposure to riociguat in patients with $\mathrm{PH}$ compared with those without $\mathrm{PH}$, individual dose titration should allow riociguat therapy to be tailored to the individual according to blood pressure and clinical response. Patients should be started on riociguat $1.0 \mathrm{mg}$ three times daily $(0.5 \mathrm{mg}$ three times daily in patients who may not tolerate hypotensive effects), and-at intervals of at 
least 2 weeks - the dose increased by $0.5 \mathrm{mg}$ three times daily to a maximum of $2.5 \mathrm{mg}$ three times daily; however, if blood pressure falls below $95 \mathrm{mmHg}$ the dose should not be uptitrated, and if the patient develops hypotension the dose should be decreased by $0.5 \mathrm{mg}$ three times daily. ${ }^{12,24}$

The pharmacodynamic effects of a single dose of riociguat $1.0 \mathrm{mg}$ that were observed in this study are not necessarily predictive of the pharmacodynamic effects of riociguat when used as chronic treatment in patients with PAH or CTEPH. However, the pharmacokinetic results are in line with those of multiple-dose studies in healthy individuals and in patients. ${ }^{10,11,38,39}$ This plus the knowledge that riociguat displays dose-proportional and linear kinetics and that a single dose of riociguat is pharmacologically effective ${ }^{8}$ mean that the pharmacokinetic conclusions from this single-dose study could potentially be applied to multiple-dosing steady-state conditions.

Our study should be considered in the context of other investigations from our group of the interactions between riociguat and a spectrum of commonly used drugs, including aspirin, ketoconazole, clarithromycin, and omeprazole. ${ }^{40-45}$ The present study adds to current knowledge by demonstrating that riociguat exposure in patients with moderate hepatic impairment is elevated compared with that in age-, weight-, and sex-matched controls even though the level of exposure overlaps with that previously reported in healthy volunteers. Individual dose titration and close monitoring of blood pressure and clinical well-being, as performed in the phase 3 trials $^{10,11}$ and recommended in European and US labeling, ${ }^{12,24}$ should overcome this risk of increased exposure. In summary, moderate liver impairment (Child-Pugh B hepatic impairment) results in increased exposure to riociguat compared with that in controls, and particular care should be exercised during individual dose titration when prescribing the drug to patients with this condition.

\section{ACKNOWLEDGMENTS}

The studies were performed by Dr. Atef Halabi, Medical Director, CRS Clinical Research Services, Kiel, Lornsenstrasse 7, 24105 Kiel, Germany. Selected data included in this article have been presented at the American Thoracic Society International Conference, Philadelphia, Pennsylvania, May 17-22, 2013, and at the 6th International Conference on cGMP, Erfurt, Germany, June 28-30, 2013. ${ }^{46}$

Source of Support: The study was funded by a research grant from Bayer Pharma. Medical writing support and editorial assistance were provided by Dr. Bernd Sierakowski of Sierakowski Medical Writing and by Oxford PharmaGenesis (Oxford, United Kingdom) and were funded by Bayer Pharma.

Conflict of Interest: All authors are employees of Bayer Pharma. In addition, RF, WM, AS, SU, and GW have stock in Bayer but are not paid in stock or stock options.

\section{REFERENCES}

1. D’Alonzo GE, Barst RJ, Ayres SM, et al. Survival in patients with primary pulmonary hypertension: results from a national prospective registry. Ann Intern Med 1991;115:343-349.

2. Benza RL, Miller DP, Barst RJ, Badesch DB, Frost AE, McGoon MD. An evaluation of long-term survival from time of diagnosis in pulmo- nary arterial hypertension from the REVEAL registry. Chest 2012; 142:448-456.

3. Evgenov OV, Pacher P, Schmidt PM, Hasko G, Schmidt HH, Stasch JP. NO-independent stimulators and activators of soluble guanylate cyclase: discovery and therapeutic potential. Nat Rev Drug Discov 2006; 5:755-768.

4. McLaughlin VV, McGoon MD. Pulmonary arterial hypertension. Circulation 2006;114:1417-1431.

5. Arnold WP, Mittal CK, Katsuki S, Murad F. Nitric oxide activates guanylate cyclase and increases guanosine $3^{\prime}: 5^{\prime}$-cyclic monophosphate levels in various tissue preparations. Proc Natl Acad Sci USA 1977;74: 3203-3207.

6. Schermuly RT, Stasch JP, Pullamsetti SS, et al. Expression and function of soluble guanylate cyclase in pulmonary arterial hypertension. Eur Respir J 2008;32:881-891.

7. Stasch JP, Pacher P, Evgenov OV. Soluble guanylate cyclase as an emerging therapeutic target in cardiopulmonary disease. Circulation 2011;123: 2263-2273.

8. Frey R, Mück W, Unger S, Artmeier-Brandt U, Weimann G, Wensing G. Single-dose pharmacokinetics, pharmacodynamics, tolerability, and safety of the soluble guanylate cyclase stimulator BAY 63-2521: an ascending-dose study in healthy male volunteers. J Clin Pharmacol 2008;48:926-934.

9. Grimminger F, Weimann G, Frey R, et al. First acute haemodynamic study of soluble guanylate cyclase stimulator riociguat in pulmonary hypertension. Eur Respir J 2009;33:785-792.

10. Ghofrani H-A, D'Armini AM, Grimminger F, et al. Riociguat for the treatment of chronic thromboembolic pulmonary hypertension. N Engl J Med 2013;369:319-329.

11. Ghofrani H-A, Galiè N, Grimminger F, et al. Riociguat for the treatment of pulmonary arterial hypertension. N Engl J Med 2013;369:330340 .

12. Bayer HealthCare Pharmaceuticals. Prescribing information [Adempas (riociguat) tablets]. Revised September 2014. http://labeling.bayerhealthcare .com/html/products/pi/Adempas_PI.pdf.

13. Walsh AA, Szklarz GD, Scott EE. Human cytochrome P450 1A1 structure and utility in understanding drug and xenobiotic metabolism. J Biol Chem 2013;288:12932-12943.

14. Czekaj P, Wiaderkiewicz A, Florek E, Wiaderkiewicz R. Tobacco smokedependent changes in cytochrome P450 1A1, 1A2, and 2E1 protein expressions in fetuses, newborns, pregnant rats, and human placenta. Arch Toxicol 2005;79:13-24.

15. McLemore TL, Adelberg S, Liu MC, et al. Expression of CYP1A1 gene in patients with lung cancer: evidence for cigarette smoke-induced gene expression in normal lung tissue and for altered gene regulation in primary pulmonary carcinomas. J Natl Cancer Inst 1990;82:1333-1339.

16. Committee for Medicinal Products for Human Use. CHMP assessment report: Adempas. London: European Medicines Agency, 2014.

17. Child C, Turcotte J, eds. Surgery and portal hypertension. Philadelphia: Saunders, 1964.

18. Pugh RN, Murray-Lyon IM, Dawson JL, Pietroni MC, Williams R. Transection of the oesophagus for bleeding oesophageal varices. $\mathrm{Br} \mathrm{J}$ Surg 1973;60:646-649.

19. European Medicines Agency. Guideline on the evaluation of the pharmacokinetics of medicinal products in patients with impaired hepatic function, 2005. Accessed February 21, 2013. http://www.ema.europa.eu /docs/en_GB/document_library/Scientific_guideline/2009/09/WC500003123 .pdf.

20. US Food and Drug Administration. Guidance for industry: pharmacokinetics in patients with impaired hepatic function-study design, data analysis, and impact on dosing and labeling. Published May 2003. Accessed February 21, 2013. http://www.fda.gov/downloads/drugs/guidancecompliance regulatoryinformation/guidances/ucm072123.pdf.

21. Ferenci P, Lockwood A, Mullen K, Tarter R, Weissenborn K, Blei AT. Hepatic encephalopathy - definition, nomenclature, diagnosis, and quan- 
tification: final report of the working party at the 11th World Congresses of Gastroenterology, Vienna, 1998. Hepatology 2002;35:716-721.

22. Cockcroft DW, Gault MH. Prediction of creatinine clearance from serum creatinine. Nephron 1976;16:31-41.

23. Schuhmacher J, Buhner K, Witt-Laido A. Determination of the free fraction and relative free fraction of drugs strongly bound to plasma proteins. J Pharm Sci 2000;89:1008-1021.

24. European Medicines Agency. Annex I: summary of product characteristics [Adempas (riociguat tablets)]. Accessed November 14, 2014. http://ec .europa.eu/health/documents/community-register/2014/20140327128191 /anx_128191_en.pdf.

25. European Medicines Agency. Adempas European Public Assessment Report: product information 2014. Accessed August 15, 2014. http://www .ema.europa.eu/ema/index.jsp?curl=pages/medicines/human/medicines 1002737/human_med_001733.jsp\&mid=WC0b01ac058001d124.

26. Schuppan D, Afdhal NH. Liver cirrhosis. Lancet 2008;371:838-851.

27. Shimada T, Yamazaki H, Mimura M, et al. Characterization of microsomal cytochrome P450 enzymes involved in the oxidation of xenobiotic chemicals in human fetal liver and adult lungs. Drug Metab Dispos 1996;24:515-522.

28. Wei C, Caccavale RJ, Kehoe JJ, Thomas PE, Iba MM. CYP1A2 is expressed along with CYP1A1 in the human lung. Cancer Lett 2001;171: 113-120.

29. Anttila S, Tuominen P, Hirvonen A, et al. CYP1A1 levels in lung tissue of tobacco smokers and polymorphisms of CYP1A1 and aromatic hydrocarbon receptor. Pharmacogenetics 2001;11:501-509.

30. Drahushuk AT, McGarrigle BP, Larsen KE, Stegeman JJ, Olson JR. Detection of CYP1A1 protein in human liver and induction by TCDD in precision-cut liver slices incubated in dynamic organ culture. Carcinogenesis 1998;19:1361-1368.

31. Nishimura M, Yaguti H, Yoshitsugu H, Naito S, Satoh T. Tissue distribution of mRNA expression of human cytochrome P450 isoforms assessed by high-sensitivity real-time reverse transcription PCR. Yakugaku Zasshi 2003;123:369-375.

32. Whitlock JP Jr. Induction of cytochrome P4501A1. Annu Rev Pharmacol Toxicol 1999;39:103-125.

33. Kim JH, Sherman ME, Curriero FC, Guengerich FP, Strickland PT, Sutter TR. Expression of cytochromes P450 1A1 and 1B1 in human lung from smokers, non-smokers, and ex-smokers. Toxicol Appl Pharmacol 2004;199:210-219.

34. Frey R, Becker C, Unger S, Schmidt A, Wensing G, Mueck W. Pharmacokinetics of the soluble guanylate cyclase stimulator riociguat in individuals with renal impairment. BMC Pharmacol Toxicol 2013;14 (suppl. 1):P22.

35. Bayer HealthCare Pharmaceuticals. Briefing document for Cardiovascular and Renal Drugs Advisory Committee: riociguat (BAY 63-2521). Published August 6, 2013. http://www.fda.gov/downloads/advisorycommittees /committeesmeetingmaterials/drugs/cardiovascularandrenaldrugsadvisory committee/ucm363543.pdf.

36. Alvarez AM, Mukherjee D. Liver abnormalities in cardiac diseases and heart failure. Int J Angiol 2011;20:135-142.

37. Mielniczuk LM, Chandy G, Stewart D, et al. Worsening renal function and prognosis in pulmonary hypertension patients hospitalized for right heart failure. Congest Heart Fail 2012;18:151-157.

38. Ghofrani HA, Hoeper MM, Halank M, et al. Riociguat for chronic thromboembolic pulmonary hypertension and pulmonary arterial hypertension: a phase II study. Eur Respir J 2010;36:792-799.

39. Frey R, Mück W, Kirschbaum N, Krätzschmar J, Weimann G, Wensing G. Riociguat (BAY 63-2521) and warfarin: a pharmacodynamic and pharmacokinetic interaction study. J Clin Pharmacol 2011;51:10511060 .

40. Becker C, Frey R, Unger S, Artmeier-Brandt U, Weimann G, Mück $\mathrm{W}$. Effects of omeprazole and aluminum hydroxide/magnesium hydroxide on riociguat absorption. Pulm Circ 2016;6(S1):S43-S48.

41. Becker C, Frey R, Unger S, et al. Pharmacokinetic interaction of ketoconazole, clarithromycin, and midazolam with riociguat. BMC Pharmacol Toxicol 2013;14(suppl. 1):P5

42. Frey R, Mueck W, Unger S, et al. No pharmacodynamic (PD) and pharmacokinetic (PK) interaction of riociguat (BAY 63-2521) and aspirin. BMC Pharmacol Toxicol 2011;11(suppl. 1):P25.

43. Becker C, Frey R, Unger S, Artmeier-Brandt U, Weimann G, Mueck W. Effects of omeprazole and $\mathrm{AlOH} / \mathrm{MgOH}$ on riociguat absorption. BMC Pharmacol Toxicol 2013;14(suppl. 1):P6.

44. Becker C, Frey R, Unger S, Thomas D, Reber M, Weimann G, Dietrich $\mathrm{H}$, Arens ER, Mück W. Pharmacokinetic interaction of riociguat with ketoconazole, clarithromycin, and midazolam. Pulm Circ 2016:6(S1): S49-S57.

45. Frey R, Reber M, Krätzschmar J, Unger S, Mück W, Wensing G. Riociguat (BAY 63-2521) and aspirin: a randomized, pharmacodynamic, and pharmacokinetic interaction study. Pulm Circ 2016:6(S1):S35-S42.

46. Frey R, Becker C, Unger S, Schmidt A, Wensing G, Mueck W. Pharmacokinetics of the soluble guanylate cyclase stimulator riociguat in individuals with hepatic impairment. BMC Pharmacol Toxicol 2013;14 (suppl. 1):P21. 